\title{
DNA vaccine encoding human papillomavirus antigens flanked by a signal peptide and a KDEL sequence induces a potent therapeutic antitumor effect
}

\author{
JOSE J. PEREZ-TRUJILLO ${ }^{1}$, RODOLFO GARZA-MORALES ${ }^{1}$, JOSE A. BARRON-CANTU ${ }^{1}$, \\ GABRIEL FIGUEROA-PARRA ${ }^{1}$, ARACELY GARCIA-GARCIA ${ }^{1}$, HUMBERTO RODRIGUEZ-ROCHA ${ }^{1}$, \\ JAIME GARCIA-JUAREZ ${ }^{1}$, GERARDO E. MUÑOZ-MALDONADO ${ }^{2}$, ODILA SAUCEDO-CARDENAS ${ }^{1,3}$, \\ ROBERTO MONTES-DE-OCA-LUNA ${ }^{1}$ and MARIA DE JESUS LOERA-ARIAS ${ }^{1}$ \\ ${ }^{1}$ Department of Histology, Faculty of Medicine, Universidad Autonoma de Nuevo Leon; \\ ${ }^{2}$ General Surgery Service, University Hospital ‘Dr Jose Eleuterio Gonzalez', Universidad Autonoma de Nuevo Leon, \\ Monterrey, Nuevo Leon 64460; ${ }^{3}$ Division of Genetics, Northeast Biomedical Research Center, \\ Instituto Mexicano del Seguro Social, Monterrey, Nuevo Leon 64720, México
}

Received July 7, 2015; Accepted October 13, 2016

DOI: $10.3892 / \mathrm{ol} .2017 .5635$

\begin{abstract}
Cellular immune responses play a critical role in the eradication of intracellular infections and malignant cells through the recognition and subsequent removal of the infection or malignant cells. Effective antigen presentation is crucial for stimulating the immune system against malignant cells. Calreticulin (CRT) has been used to improve antigen presentation. However, CRT overexpression has been previously associated with the development of pancreatic and breast cancer. The import and retention signals of CRT in the endoplasmic reticulum (ER) can be used to overcome CRT overexpression. The present study describes the potent antitumor effect of a DNA vaccine encoding human papillomavirus type 16 E6 and E7 antigens flanked by ER import and retention signals (SP-E6E7m-KDEL). The effect of this vaccine was compared with that of E6 and E7 antigens fused to human full-length CRT (hCRT-E6E7m). In the present
\end{abstract}

Correspondence to: Dr Maria de Jesus Loera-Arias, Department of Histology, Faculty of Medicine, Universidad Autonoma de Nuevo Leon, Madero y Aguirre Pequeno s/n Mitras Centro, Monterrey, Nuevo Leon 64460, México

E-mail: loera.arias@gmail.com

Abbreviations: CRT, calreticulin; DAPI, 4',6-diamidino-2phenylindole; ER, endoplasmic reticulum; H\&E, hematoxylin and eosin; hCRT, human calreticulin; HEK-293, human embryonic kidney 293 cells; HPV, human papillomavirus; IFN- $\gamma$, interferon $\gamma$; KDEL, lysine-aspartic acid-glutamic acid-leucine peptide sequence; MHC-I, major histocompatibility complex I; rCRT, rabbit calreticulin; SP, signal peptide; TAPs, transporters associated with antigen processing proteins

Key words: antigen targeting, calreticulin, DNA vaccination, E6, E7, endoplasmic reticulum, HPV16, KDEL sequence, signal peptide study, the effectiveness of SP-E6E7m-KDEL for inducing an interferon- $\gamma$ antigen-specific, response and its therapeutic effect against tumors was demonstrated, which was as effective as immunization against those antigens fused to CRT. This simplified strategy, using ER import and retention signal peptides to direct antigens to this organelle, provides an efficient alternative to traditional vaccines and, more importantly, a safe and potent system to induce a therapeutic antitumor response.

\section{Introduction}

Cervical cancer arises from viral infections and is particularly associated with persistent human papillomavirus (HPV) infection. As a result, the tumor cells themselves express virus-specific antigens, including the oncoproteins E6 and E7, which are involved in cellular malignant transformation (1).

Cluster of differentiation $8\left(\mathrm{CD}^{+}\right)$cytotoxic lymphocytes are the primary effector cells in cell-mediated immunity, and the activation of these cells is required for the recognition of the epitope-loaded major histocompatibility complex I (MHC-I) of the target cell (2). These epitopes are typically produced by proteasomal protein degradation and are subsequently carried by transporters associated with antigen processing proteins (TAPs), for entry into the endoplasmic reticulum (ER) and loading onto MHC-I (3).

Only a single peptide is loaded onto the MHC-I for every $10^{4}$ degraded proteins (4). In tumors or virus-infected cells, MHC-I expression may be affected by a reduction in antigen presentation, leading to immune system evasion $(5,6)$. In previous years, various strategies, involving DNA and adenovirus vaccines, have been designed to overcome this limitation and to potentiate the antitumor immune response (7-9). A promising strategy is based on targeted antigen delivery directly to the ER, through the fusion of these proteins to an ER resident protein or an ER signal peptide. Previous studies have shown that the fusion of antigens with ER resident 
proteins, particularly calreticulin (CRT), enhances the immune response compared with antigens alone $(10,11)$. However, CRT overexpression has recently been associated with pancreatic cancer through the mitogen activated protein kinase/extracellular signal-regulated kinase signaling pathway (12) and breast cancer through elevated humoral immunity to CRT (13).

In a previous study using mice, we demonstrated that treatment with an adenovirus (Ad SP-E7-KDEL) expressing antigen E7 fused to a CRT signal peptide (SP: MLLPVPLLLGLLGLAAAL) and a retention peptide (KDEL) exerted a protective antitumor effect equal to that obtained following treatment with the same antigen fused to CRT (14). In the present study, an improved vaccine was constructed that includes the E6 and E7 antigens, which provides a better antitumor effect according to the literature (15-17). In addition, the two antigens were mutant versions to eliminate the risk of cellular transformation $(18,19)$ and were codon-optimized for efficient expression in mammalian cells (20). These antigens were fused to the human CRT signal peptide and the KDEL signal (SP-E6E7m-KDEL). The effect of this construct was compared with that obtained by the fusion of these antigens to human full-length CRT (hCRT-E6E7m); as a reference control, rabbit CRT (rCRT-E7) was fused to wild-type E7 (10). These constructs were evaluated as DNA vaccines via gene gun-mediated transfer.

\section{Materials and methods}

Mice. Female C57BL/6 mice (6-8 weeks old) were purchased from Harlan (Mexico City, Mexico), housed under a $12 \mathrm{~h}$ light/12 h dark cycle and provided with ad libitum access to food and water. The experiments reported in the present study were conducted according to the principles set forth in the Guide for the Care and Use of Laboratory Animals of the U.S. National Institutes of Health. The protocol was approved by the Ethics Committee of the School of Medicine, Universidad Autonoma de Nuevo Leon (Monterrey, Mexico) (protocol HT14-002).

Cell lines. HEK-293 human embryonic kidney (\#CRL-1573) and TC-1 mouse lung tumor (\#CRL-2785) cell lines were obtained from the American Type Culture Collection (Manassas, VA, USA). HEK-293 cells were cultured in advanced Dulbecco's modified Eagle medium supplemented with $4 \%$ heat-inactivated fetal calf serum, 2 mM L-glutamine and $100 \mathrm{U} / \mathrm{ml}$ penicillin/streptomycin (all from Cellgro, Mediatech Inc., Manassas, VA, USA). TC-1 cells were cultured in RPMI 1640 medium supplemented with $10 \%$ heat-inactivated fetal calf serum, $1 \mathrm{mM}$ sodium pyruvate (Thermo Fisher Scientific, Inc., Waltham, MA, USA), $100 \mathrm{U} / \mathrm{ml}$ penicillin/streptomycin and G418 at $0.5 \mathrm{mg} / \mathrm{ml}$ (\#A1720; Sigma-Aldrich; Merck Millipore, Darmstadt, Germany). All cells were maintained at $37^{\circ} \mathrm{C}$ in a $5 \% \mathrm{CO}_{2}$ atmosphere.

DNA constructs. The sequences of the fusion proteins SP-E6E7m-KDEL and hCRT-E6E7m were designed in silico and synthesized at Eurofins Company (Huntsville, AL, USA). These genes were subcloned using the NotI and BglII restriction sites in the pShuttle-CMV vector and purified using an endotoxin-free kit (Qiagen, Inc., Valencia, CA, USA). The plasmid pShuttle:rCRT-E7 was donated by Dr Jorge
Gomez-Gutierrez from the Division of Surgical Oncology of the University of Louisville (Louisville, KY, USA) (11). DNA cartridges ( $1 \mu \mathrm{g} / \mathrm{shot})$ for the Helios gene gun system (Bio-Rad Laboratories, Inc., Hercules, CA, USA) were prepared according to the manufacturer's protocol and stored in desiccant chambers at $4^{\circ} \mathrm{C}$ until further use.

Western blot analysis. A total of $3 \times 10^{5}$ HEK-293 cells were seeded onto a 6 -well plate. The next day, $4 \mu \mathrm{g}$ of the corresponding DNA was mixed with Turbofect (\#R0531; Thermo Fisher Scientific, Inc.) and the cells were transfected according to the manufacturer's protocol. After $18 \mathrm{~h}$, the cells were collected and lysed with radioimmunoprecipitation assay (RIPA) buffer. The supernatants were mixed with loading buffer, denatured at $100^{\circ} \mathrm{C}$ for $10 \mathrm{~min}$, and centrifuged at $18,000 \mathrm{x} g$. The samples were subjected to denaturing electrophoresis on $12 \%$ acrylamide gels and subsequently transferred to nitrocellulose membranes. The membranes were blocked for $1 \mathrm{~h}$ with $3 \%$ bovine serum albumin (\#A1311;US Biological, Salem, MA, USA) in Tris-buffered saline and Tween 20 (TBST) (135 mM NaCl, $2.7 \mathrm{mM} \mathrm{KCl}$, $24.8 \mathrm{mM}$ Tris- $\mathrm{HCl}, 0.05 \%$ Tween $20, \mathrm{pH} 7.4)$. Similarly, the antibodies were diluted with $0.3 \%$ BSA in TBST. Subsequently, the membranes were incubated overnight with the mouse monoclonal IgG2a raised against amino acids 35-56 of E7 HPV16 (\#sc-65711; Santa Cruz Biotechnology, Inc., Dallas, TX, USA) diluted 1:3,000. The membranes were washed with TBST and incubated for $2 \mathrm{~h}$ with a horseradish peroxidase (HRP)-conjugated rabbit anti-mouse antibody (\#A9044; Sigma-Aldrich; Merck Millipore) diluted 1:10,000. The signal detection was performed using Super Signal West Pico Chemiluminescent Substrate (\#34080; Pierce Biotechnology; Thermo Fisher Scientific, Inc.). The same membrane was washed with Restore Western Blot Stripping Buffer (\#21063; Thermo Fisher Scientific, Inc.) and incubated with the mouse monoclonal $\mathrm{IgG}_{1}$ raised against HPV16/18 E6 protein (\#sc-460; Santa Cruz Biotechnology, Inc.) diluted 1:3,000.

Immunofluorescence. Glass coverslips were sterilized and placed into the wells of a 24 -well plate. Subsequently, $5 \times 10^{4}$ HEK-293 cells were seeded into the wells of the prepared plates and transfected the next day with $1 \mu \mathrm{g}$ of DNA using Turbofect (\#R0531; Fermentas; Thermo Fisher Scientific, Inc.). After $16 \mathrm{~h}$, the cells were fixed with methanol:acetone (1:1), blocked with 3\% horse serum (\#16050122; Thermo Fisher Scientific, Inc) and incubated overnight with mouse monoclonal IgG2a raised against amino acids 35-56 of E7 HPV16 and rabbit polyclonal raised against amino acids 1-70 of human calnexin, diluted 1:250 each one (\#sc-65711 and \#sc-11397; Santa Cruz Biotechnology, Inc.). The membranes were then washed with TBST and incubated for $2 \mathrm{~h}$ with goat polyclonal anti-mouse IgG $(\mathrm{H}+\mathrm{L})$ Alexa 594 and goat polyclonal anti-rabbit IgG (H+L) Alexa 488 (\#A-11005 and \#A-11034; Invitrogen; Thermo Fisher Scientific, Inc.) each diluted 1:500. The glass coverslips were mounted with Vectashield Antifade Mounting Medium with 4',6-diamidino-2-phenylindole (DAPI) (\#H-1200; Vector Laboratories, Inc., Burlingame, CA, USA) and observed using an Apotome fluorescence microscope (Carl Zeiss, Inc., Thornwood, NY, USA). 
Interferon (IFN)- $\gamma$ quantification. Groups of 3 mice (6-7 weeks old) were immunized on days 0 and 7 with $1 \mu \mathrm{g}$ of DNA in the abdominal area using the gene gun system. The mice were sacrificed 7 days subsequent to immunization, and the spleens were collected. A pool of spleens for each treatment was mashed on a cell strainer (\#352350; BD Falcon, Bedford, MA, USA), and the splenocytes were stimulated with $1 \mu \mathrm{g} / \mathrm{ml}$ E7 immunodominant epitope (RAHYNIVTF; amino acids 49-57) or E6 (YDFAFRDL; amino acids 50-57) (both from GenWay Biotech, Inc., San Diego, CA USA) for $48 \mathrm{~h}$. The culture supernatants were collected, and IFN- $\gamma$ levels were determined using a mouse IFN- $\gamma$ platinum enzyme linked immunosorbent assay (ELISA) kit (\#BMS606; eBioscience, Inc., San Diego, CA, USA). The basal levels of IFN- $\gamma$ produced under non-antigen stimulation conditions were used to normalize the results.

Antitumor therapeutic assay. Groups of 5 mice were used for each treatment. The mice were injected with $1 \times 10^{4}$ TC-1 cells in the tail vein, simulating a metastasis model. When this lung tumor cell line is injected in the tail vein, the first capillary bed that cells face is in the lungs, and therefore all cells are retained and grow on lung epithelium. The gene gun system was used for immunization in the abdominal area using $300 \mathrm{psi}$ as output pressure. The mice were immunized with $1 \mu \mathrm{g}$ of the corresponding DNA construct on the third and tenth days following tumor implantation and were sacrificed on day 25. The lungs were rinsed in phosphate buffered saline (PBS) and fixed in 4\% paraformaldehyde (PFA) in PBS for tumor foci counts.

Histological analysis of the tumor foci. After counting the tumor foci, lungs were fixed in 4\% PFA-PBS, dehydrated and embedded in paraffin. Lung orientation was disposed, prioritizing areas with more tumor foci; $4 \mu \mathrm{m}$ sections were deparaffinized and hydrated for hematoxylin and eosin (H\&E) staining (H3136 and E6003; Sigma-Aldrich; Merck Millipore), followed by dehydration and mounting using Entellan mounting medium (\#17960; Merck Millipore). The lung sections were analyzed using a Primo Star microscope (Carl Zeiss, Inc., Thornwood, NY, USA), the images were obtained by bright-field microscopy.

Statistical analysis. Analysis of variance (one-way ANOVA) followed by post hoc Dunnett's tests was performed using Prism software (GraphPad Software, Inc., La Jolla, CA, USA). $\mathrm{P}<0.05$ was considered to indicate a statistically significant difference. All experiments were performed at least twice.

\section{Results}

Design and characterization of DNA constructs. The construct SP-E6E7m-KDEL consists of the fusion of full-length E6-E7 mutant antigens to the signal peptide of hCRT at the $\mathrm{N}$-terminus and the fusion of the KDEL signal to the C-terminus for retention in the ER. The construct hCRT-E6E7m consists of the fusion of full-length E6-E7 mutant antigens to the N-terminus of hCRT. The resulting synthetic constructs were deposited in the GenBank database under the accession numbers KP898251 and KP898250, respectively. The DNA constructs are shown in Fig. 1.

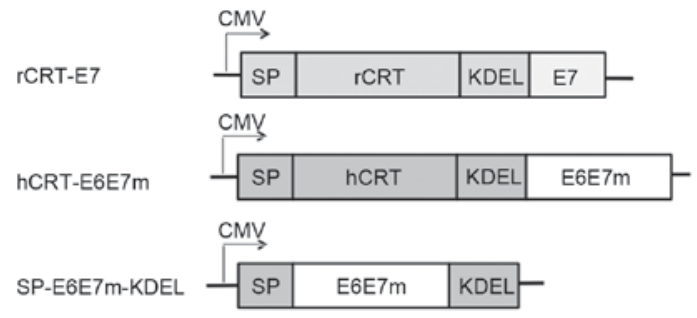

Figure 1. Construction of DNA vaccines. These gene constructs involve the fusion of the human papillomavirus $16 \mathrm{E} 6$ and E7 proteins to different signal sequences. rCRT, rabbit calreticulin; hCRT, human calreticulin, KDEL, lysine-aspartic acid-glutamic acid-leucine peptide sequence; SP, signal peptide.

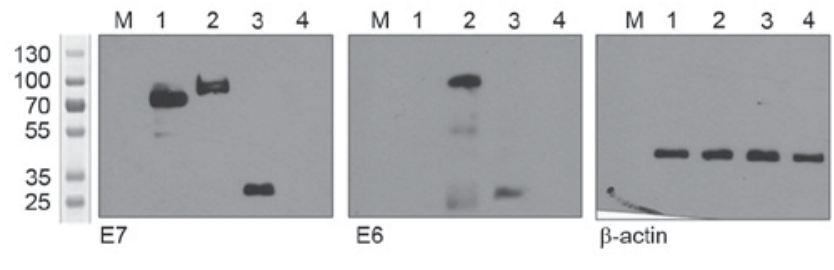

Figure 2. Detection of the recombinant proteins through western blotting. HEK-293 cells transfected with constructs expressing rCRT-E7, hCRT-E6E7m, SP-E6E7m-KDEL or the empty vector. After $24 \mathrm{~h}$, the cell protein extracts were subjected to SDS-PAGE and incubated with specific antibodies. Lane 1, rCRT-E7; Lane 2, hCRT-E6E7m; Lane 3, SP-E6E7m-KDEL; Lane 4, empty vector. A molecular-weight size marker was loaded on lane M. rCRT, rabbit calreticulin; hCRT, human calreticulin, KDEL, lysine-aspartic acid-glutamic acid-leucine peptide sequence; SP, signal peptide.

Protein extracts from transfected HEK-293 cells transfected with the different constructs were analyzed through western blotting (Fig. 2). Bands were observed at $70 \mathrm{kDa}$ for rCRT-E7, $80 \mathrm{kDa}$ for hCRT-E6E7m and $25 \mathrm{kDa}$ for SP-E6E7m-KDEL, confirming the correct expression of the recombinant proteins containing E6 and E7 from the different constructs.

Fusion to hCRT or the targeting/retention signal peptides targets the E6-E7 antigens to the ER. To determine whether the recombinant proteins are directed to the ER, HEK-293 cells were transfected with the DNA constructs and prepared for immunofluorescence. Co-localization was detected between the E7 signal (red) and the calnexin signal (green), a well-characterized resident protein of the endoplasmic reticulum (Fig. 3). This result demonstrated that antigens fused to rCRT, hCRT or targeting/retention signal peptides are directed to the ER.

Immunization with SP-E6E7m-KDEL induces IFN- $\gamma$ production. To determine whether hCRT-E6E7m and SP-E6E7m-KDEL induce an antigen-specific IFN- $\gamma$-mediated response, the mice were immunized with each DNA construct, as previously described. After 1 week, the spleens were harvested, and the splenocytes were isolated and subsequently stimulated in vitro with the E7 or E6 epitopes for $48 \mathrm{~h}$. The supernatant was collected and then analyzed by ELISA. The results show that splenocytes from the hCRT-E6E7m group had high IFN- $\gamma$ production when stimulated with E7 $(\mathrm{P}=0.007)$; however, most importantly, splenocytes from the SP-E6E7m-KDEL group that were stimulated with E6 or 


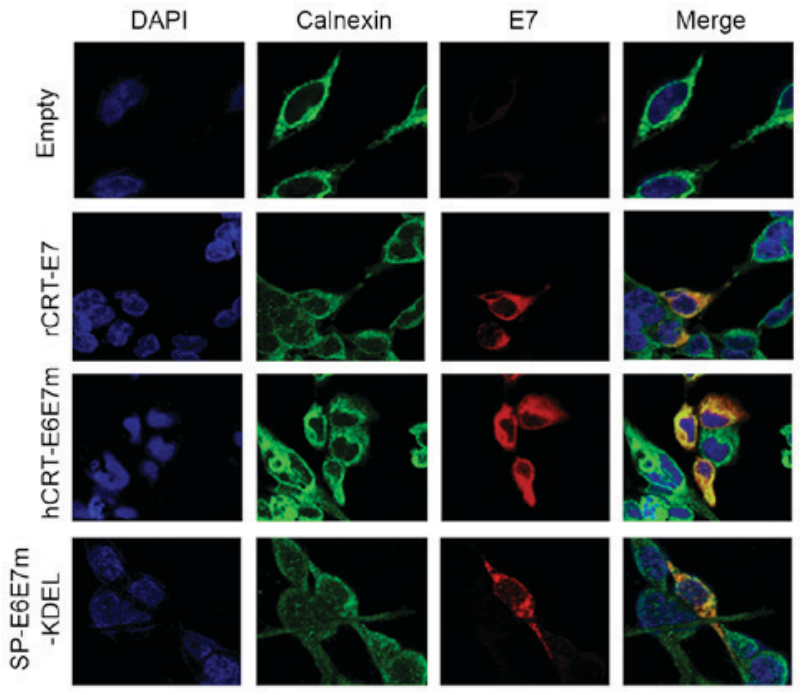

Figure 3. Subcellular localization of recombinant proteins. HEK-293 cells were transfected with constructs expressing rCRT-E7, hCRT-E6E7m, SP-E6E7m-KDEL or the empty vector. After $24 \mathrm{~h}$, the cells were fixed and incubated with specific primary antibodies and secondary antibodies conjugated with fluorochromes. The samples were examined using a confocal fluorescence microscope. Original magnification, x630. rCRT, rabbit calreticulin; hCRT, human calreticulin, KDEL, lysine-aspartic acid-glutamic acid-leucine peptide sequence; SP, signal peptide; DAPI, 4',6-diamidino-2-phenylindole.

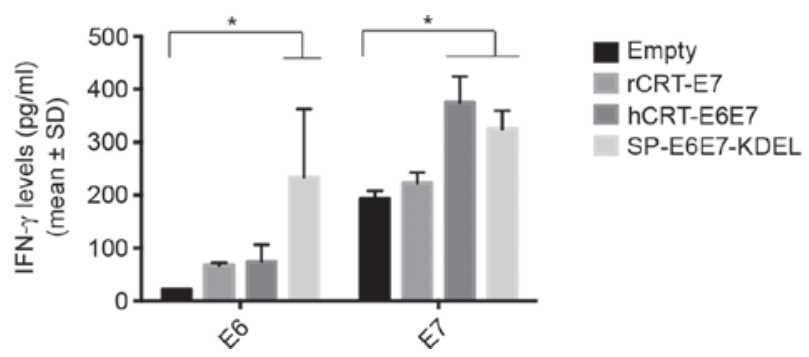

Figure 4. Enzyme linked immunosorbent assay detection of IFN- $\gamma$ production by human papillomavirus E7/E6-specific splenocytes. Groups of three mice (6-8 weeks old) were immunized in the abdominal area with $1 \mu \mathrm{g}$ of DNA on days 0 and 7 using a gene gun system. After 7 days, the splenocytes were harvested and stimulated in vitro with E7 or E6 peptide for $48 \mathrm{~h}$ The culture supernatants were collected, and IFN- $\gamma$ expression levels were determined. "P<0.05. rCRT, rabbit calreticulin; hCRT, human calreticulin, KDEL, lysine-aspartic acid-glutamic acid-leucine peptide sequence; IFN- $\gamma$, interferon- $\gamma ; \mathrm{SD}$, standard deviation.

E7 antigens had increased IFN- $\gamma$ production $(\mathrm{P}=0.021$ and $\mathrm{P}=0.017$, respectively). This suggests that targeting these antigens to the ER promotes an antigen-specific response mediated by IFN- $\gamma$ (Fig. 4).

Treatment with SP-E6E7m-KDEL induces the reduction of TC-1 tumor pulmonary nodules in C57BL/6 mice. A therapeutic antitumor effect was demonstrated in vivo. TC-1 tumor cells were implanted in mice C57BL/6 through intravenous (IV) injection. This IV implantation serves as a metastasis model to disseminate the tumor cells through the organism, although the implanted cells are predominately detected on the lungs, forming multiple nodules on the epithelium. The mice were subjected to the antitumor therapeutic assay as described above, and were sacrificed at 25 days following

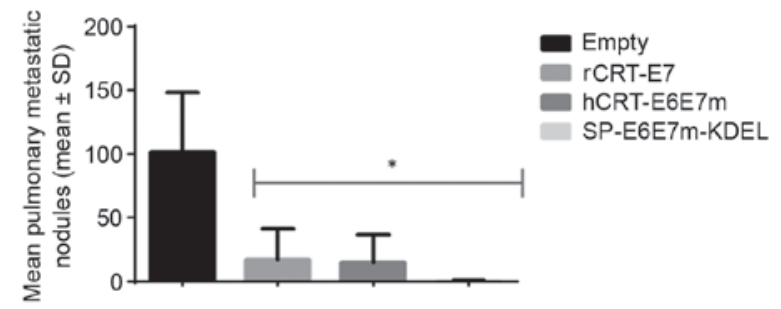

Figure 5. Antitumor effects of therapeutic DNA vaccination. Groups of 5 mice were injected with $1 \times 10^{4} \mathrm{TC}-1$ cells in the tail vein. The mice were immunized with $1 \mu \mathrm{g}$ of the corresponding DNA constructs using a gene gun system on the third and tenth days following tumor implantation and were subsequently sacrificed on day 25 . The lungs were fixed with $4 \%$ paraformaldehyde, and tumor foci present on the epithelium were counted. The values and bars represent the means and standard deviations of tumor foci, respectively. ${ }^{*} \mathrm{P}<0.05$ (rCRT-E7, hCRT-E6E7m and SP-E6E7m-KDEL vs. 'empty' control). rCRT, rabbit calreticulin; hCRT, human calreticulin, KDEL, lysine-aspartic acid-glutamic acid-leucine peptide sequence; IFN- $\gamma$, interferon- $\gamma$; SD, standard deviation.

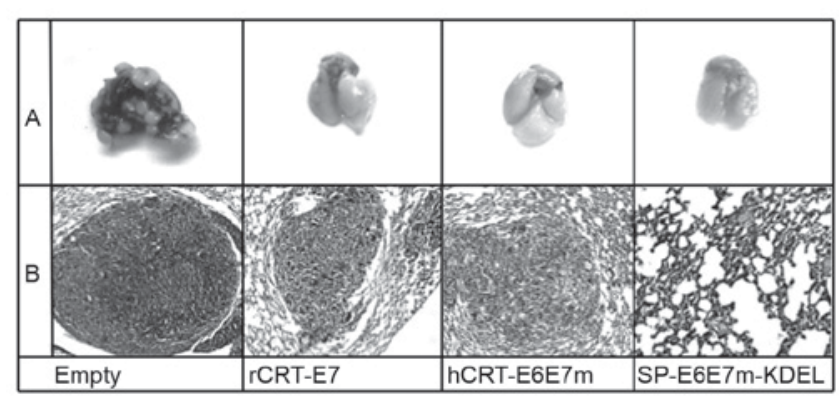

Figure 6. Histological changes in the tumor foci following therapeutic DNA vaccination. The lungs collected from the antitumor therapeutic assay were embedded in paraffin and processed for H\&E staining. These images represent general structural changes observed in the organization of tumor cells in the foci between each treatment. Magnification, x100. rCRT, rabbit calreticulin; hCRT, human calreticulin, KDEL, lysine-aspartic acid-glutamic acid-leucine peptide sequence; SP, signal peptide.

tumor implantation for the evaluation of therapeutic antitumor effects by counting the number of tumor foci between treatments (Fig. 5). Treatments with rCRT-E7, hCRT-E6E7m, and SP-E6E7m-KDEL resulted in equivalent therapeutic antitumor effects, as the number of nodules drastically decreased compared with the control $(\mathrm{P}<0.05)$, without a significant difference between each treatment $(\mathrm{P}>0.05)$. Histological sections from the lungs with the highest number of tumor nodules were stained with $\mathrm{H} \& \mathrm{E}$ to determine changes in the morphology of these tumor nodules (Fig. 6). The present study observed that the tumors from immunized mice showed certain alterations in the integrity of the capsule, as certain remnants of the extracellular matrix were detected in the tissues, suggesting increased tumor regression (21).

\section{Discussion}

CRT is a chaperone protein typically present in the ER, thereby possessing a signal peptide and the KDEL retention sequence. As a chaperone protein, CRT plays multiple roles associated with MHC-I molecule loading (22), calcium homeostasis (23) and cell migration $(24,25)$. Multiple studies have reported that the fusion of antigens to CRT and other 
chaperones (26) increases antigen-specific immune responses through cross-priming and inflammation (27-29). Although immunization with full-length CRT alone shows an antitumor effect, this protein is not as potent as the fusion of CRT with antigens (29).

Clinical studies have reported alterations in CRT expression in certain types of cancer, where overexpression has been associated with increased invasiveness, metastasis and decreased survival (30), likely reflecting the role of CRT in calcium homeostasis. Calcium mediates cell migration, and upregulation through transduction seriously affects cell invasiveness $(31,32)$.

The present study aimed to identify alternatives to the use of full-length calreticulin, in order to eliminate the overexpression of xenogeneic proteins to diminish any misbalance in homeostasis while preserving the antitumor effect (14). In the present study, we designed an improved simplified version of a DNA construct by fusing the mutant E6 and E7 antigens from HPV16 to the hCRT signal peptide and the KDEL retention sequence (SP-E6E7m-KDEL). The effects of fusion to the full-length hCRT (hCRT-E6E7m) and fusion to rCRT-E7, which was previously reported to promote antigen distribution to the ER and to enhance the antitumor response specific to this antigen (10), were compared.

In the present study, immunofluorescence analysis revealed that fusing the E6-E7 antigens to the CRT signal peptide and the KDEL retention sequence is sufficient to target these antigens to the ER, and the intracellular distribution pattern is similar to that obtained through fusion with the full-length CRT. These results are consistent with previous studies showing that the linkage of E6 and E7 to ER chaperoning proteins causes the translocation of these proteins from the nucleus to the ER $(14,26,27,29)$, resulting in an almost null signal in the nucleus.

After confirming the expression and functional targeting, the present study evaluated the antitumor effects of the constructs. Immunization by ballistic gene gun was used as a user-friendly system with the capacity to stimulate skin dendritic cells, one of the immune cell types that actively participate upon the onset of a cellular immune response (33).

To simulate an advanced state of cancer to examine these vaccines, the present study used an in vivo model consisting of administration of TC-1 tumor cells through intravenous injection to simulate a metastasis model (34). The results indicate that the simplified version of the E6-E7 antigens fused only to the signal peptide and the KDEL retention sequence from CRT significantly reduced metastases nodules compared with the control, and this antitumor effect is comparable with that obtained with the antigens fused to full-length CRT, with no significant difference between these treatments. The present study detected antigen-specific IFN- $\gamma$ production in the supernatants of cultured splenocytes, obtaining high levels of production in splenocytes from the SP-E6E7m-KDEL group. These results indicate that the antitumor effect can be achieved using only signal peptides to deliver and retain the antigens.

Although metastatic nodule reduction was observed within treatments, it will be necessary to perform an evaluation on advanced stages of cancer. Other studies of HPV vaccines have previously reported that vaccine therapies are not sufficient and require the support of chemotherapy $(35,36)$.
In summary, the present study reports a strategy for targeting antigens to the ER through fusion to the signal peptide and KDEL sequence of CRT compared with full-length CRT. The antitumor effects demonstrated using these constructs were as effective as the reference constructs, providing an alternative for vaccine design, as previously reported (14). The goal is to decrease the overexpression of proteins that may affect cellular homeostasis while also preserving the capacity of the antigen to generate an antitumor response. To the best of our knowledge, the current study is the first demonstration that a DNA vaccine encoding the antigens E6 and E7 from HPV16 fused to a signal peptide and a KDEL sequence to induce a potent therapeutic antitumor effect.

\section{Acknowledgements}

This study was supported through grants from the Program to Support Research in Science and Technology (grant no. CN1096-11) from the Universidad Autonoma de Nuevo Leon and National Council for Science and Technology (CONACYT; grant no. CB-10-158509). JJPT and RGM were recipients of scholarships from CONACYT. The authors would like to thank Dr Juan Carlos Segoviano-Ramirez from the Bioimaging Unit in the Center for Research and Development in the Health Sciences, Autonomous University of Leon for kindly providing assistance with confocal imaging.

\section{References}

1. Tan S, de Vries EG, van der Zee AG and de Jong S: Anticancer drugs aimed at E6 and E7 activity in HPV-positive cervical cancer. Curr Cancer Drug Targets 12: 170-184, 2012.

2. de Boer MA, Jordanova ES, van Poelgeest MI, van den Akker BE, van der Burg SH, Kenter GG and Fleuren GJ: Circulating human papillomavirus type 16 specific T-cells are associated with HLA Class I expression on tumor cells, but not related to the amount of viral oncogene transcripts. Int J Cancer 121: 2711-2715, 2007.

3. Bukur J, Jasinski S and Seliger B: The role of classical and non-classical HLA class I antigens in human tumors. Semin Cancer Biol 22: 350-358, 2012.

4. Yewdell JW: Not such a dismal science: The economics of protein synthesis, folding, degradation and antigen processing. Trends Cell Biol 11: 294-297, 2001.

5. Ashrafi GH, Brown DR, Fife KH and Campo MS: Down-regulation of MHC class I is a property common to papillomavirus E5 proteins. Virus Res 120: 208-211, 2006.

6. Hicklin DJ, Marincola FM and Ferrone S: HLA class I antigen downregulation in human cancers: T-cell immunotherapy revives an old story. Mol Med Today 5: 178-186, 1999.

7. Tatsis $\mathrm{N}$ and Ertl HC: Adenoviruses as vaccine vectors. Mol Ther 10: 616-629, 2004.

8. Neefjes J, Jongsma ML, Paul P and Bakke O: Towards a systems understanding of MHC class I and MHC class II antigen presentation. Nat Rev Immunol 11: 823-836, 2011.

9. Yang B, Jeang J, Yang A, Wu TC and Hung CF: DNA vaccine for cancer immunotherapy. Hum Vaccin Immunother 10: 3153-3164, 2014.

10. Hsieh CJ, Kim TW, Hung CF, Juang J, Moniz M, Boyd DA, He L, Chen PJ, Chen $\mathrm{CH}$ and Wu TC: Enhancement of vaccinia vaccine potency by linkage of tumor antigen gene to gene encoding calreticulin. Vaccine 22: 3993-4001, 2004.

11. Gomez-Gutierrez JG, Elpek KG, Montes de Oca-Luna R, Shirwan H, Sam Zhou H and McMasters KM: Vaccination with an adenoviral vector expressing calreticulin-human papillomavirus 16 E7 fusion protein eradicates E7 expressing established tumors in mice. Cancer Immunol Immunother 56: 997-1007, 2007.

12. Sheng W, Chen C, Dong M, Zhou J, Liu Q, Dong Q and Li F: Overexpression of calreticulin contributes to the development and progression of pancreatic cancer. J Cell Physiol 229: 887-897, 2014. 
13. Erić-Nikolić A, Milovanović Z, Sánchez D, Pekáriková A Džodić R, Matić IZ, Tučková L, Jevrić M, Buta M, Rašković S and Juranić Z: Overexpression of calreticulin in malignant and benign breast tumors: Relationship with humoral immunity. Oncology 82: 48-55, 2012.

14. Loera-Arias MJ, Martínez-Pérez AG, Barrera-Hernández A, Ibarra-Obregón ER, González-Saldívar G, Martínez-Ortega JI, Rosas-Taraco A, Villanueva-Olivo A, Esparza-González SC, Villatoro-Hernandez J, et al: Targeting and retention of HPV16 E7 to the endoplasmic reticulum enhances immune tumour protection. J Cell Mol Med 14: 890-894, 2010.

15. Peng S, Tomson TT, Trimble C, He L, Hung CF and Wu TC: A combination of DNA vaccines targeting human papillomavirus type 16 E6 and E7 generates potent antitumor effects. Gene Ther 13: 257-265, 2006.

16. Yan J, Reichenbach DK, Corbitt N, Hokey DA, Ramanathan MP, McKinney KA, Weiner DB and Sewell D: Induction of antitumor immunity in vivo following delivery of a novel HPV-16 DNA vaccine encoding an E6/E7 fusion antigen. Vaccine 27: 431-440, 2009.

17. Zhou X, Qian X, Zhao Q, Lu Y and Xiong M: Efficient expression of modified human papillomavirus $16 \mathrm{e} 6 / \mathrm{e} 7$ fusion protein and the antitumor efficacy in a mouse model. Biol Pharm Bull 27: 303-307, 2004

18. Shi W, Bu P, Liu J, Polack A, Fisher S and Qiao L: Human papillomavirus type 16 E7 DNA vaccine: Mutation in the open reading frame of $\mathrm{E} 7$ enhances specific cytotoxic T-lymphocyte induction and antitumor activity. J Virol 73: 7877-7881, 1999.

19. Bahrami AA, Ghaemi A, Tabarraei A, Sajadian A, Gorji A and Soleimanjahi H: DNA vaccine encoding HPV-16 E7 with mutation in L-Y-C-Y-E pRb-binding motif induces potent anti-tumor responses in mice. J Virol Methods 206: 12-18, 2014.

20. Cheung YK, Cheng SC, Sin FW and Xie Y: Plasmid encoding papillomavirus Type 16 (HPV16) DNA constructed with codon optimization improved the immunogenicity against HPV infection. Vaccine 23: 629-638, 2004.

21. Amine A, Rivera S, Opolon P, Dekkal M, Biard DS, Bouamar H, Louache F, McKay MJ, Bourhis J, Deutsch E and Vozenin-Brotons MC: Novel anti-metastatic action of cidofovir mediated by inhibition of E6/E7, CXCR4 and Rho/ROCK signaling in HPV+ tumor cells. PLoS One 4: e5018, 2009.

22. Del Cid N, Jeffery E, Rizvi SM, Stamper E, Peters LR, Brown WC, Provoda C and Raghavan M: Modes of calreticulin recruitment to the major histocompatibility complex class I assembly pathway. J Biol Chem 285: 4520-4535, 2010.

23. Gelebart P, Opas M and Michalak M: Calreticulin, a Ca2+-binding chaperone of the endoplasmic reticulum. Int J Biochem Cell Biol 37: 260-266, 2005.

24. Murphy-Ullrich JE: The de-adhesive activity of matricellular proteins: Is intermediate cell adhesion an adaptive state? J Clin Invest 107: 785-790, 2001.
25. Goicoechea S, Orr AW, Pallero MA, Eggleton P and Murphy-Ullrich JE: Thrombospondin mediates focal adhesion disassembly through interactions with cell surface calreticulin. J Biol Chem 275: 36358-36368, 2000.

26. Kim JW, Hung CF, Juang J, He L, Kim TW, Armstrong DK, Pai SI, Chen PJ, Lin CT, Boyd DA and Wu TC: Comparison of HPV DNA vaccines employing intracellular targeting strategies. Gene Ther 11: 1011-1018, 2004.

27. Peng S, Ji H, Trimble C, He L, Tsai YC, Yeatermeyer J, Boyd DA, Hung CF and Wu TC: Development of a DNA vaccine targeting human papillomavirus type 16 oncoprotein E6. J Virol 78 8468-8476, 2004.

28. Kang TH, Chung JY, Monie A, Pai SI, Hung CF and Wu TC: Enhancing DNA vaccine potency by co-administration of xenogenic MHC class-I DNA. Gene Ther 17: 531-540, 2010.

29. Cheng WF, Hung CF, Chai CY, Hsu KF, He L, Ling M and Wu TC: Tumor-specific immunity and antiangiogenesis generated by a DNA vaccine encoding calreticulin linked to a tumor antigen. J Clin Invest 108: 669-678, 2001.

30. Zamanian M, Veerakumarasivam A, Abdullah S and Rosli R: Calreticulin and cancer. Pathol Oncol Res 19: 149-154, 2013.

31. Chiang WF, Hwang TZ, Hour TC, Wang LH, Chiu CC, Chen HR, Wu YJ, Wang CC, Wang LF, Chien CY, et al: Calreticulin, an endoplasmic reticulum-resident protein, is highly expressed and essential for cell proliferation and migration in oral squamous cell carcinoma. Oral Oncol 49: 534-541, 2013.

32. Shi F, Shang L, Pan BQ, Wang XM, Jiang YY, Hao JJ, Zhang Y, Cai Y, Xu X, Zhan QM and Wang MR: Calreticulin promotes migration and invasion of esophageal cancer cells by upregulating neuropilin-1 expression via STAT5A. Clin Cancer Res 20: 6153-6162, 2014.

33. Peachman KK, Rao M and Alving CR: Immunization with DNA through the skin. Methods 31: 232-242, 2003.

34. Ji H, Chang EY, Lin KY, Kurman RJ, Pardoll DM and Wu TC: Antigen-specific immunotherapy for murine lung metastatic tumors expressing human papillomavirus type 16 E7 oncoprotein. Int J Cancer 78: 41-45, 1998.

35. Lee SY, Kang TH, Knoff J, Huang Z, Soong RS, Alvarez RD, Hung CF and Wu TC: Intratumoral injection of therapeutic HPV vaccinia vaccine following cisplatin enhances HPV-specific antitumor effects. Cancer Immunol Immunother 62: 1175-1185, 2013.

36. Chen S, Liao C, Lai Y, Fan Y, Lu G, Wang H, Zhang X, Lin MC, Leng S and Kung HF: De-oncogenic HPV E6/E7 vaccine gets enhanced antigenicity and promotes tumoricidal synergy with cisplatin. Acta Biochim Biophys Sin (Shanghai) 46: 6-14, 2014. 